\title{
Variability of User Interaction with Multi-Platform News Feeds
}

\author{
Chandresh Chhatpar \\ Conservation Management Institute \\ Virginia Tech, Blacksburg, Va 24061 \\ cchhatpa@vt.edu
}

\author{
Stelios Lambros, Manuel A. Pérez-Quiñones \\ Department of Computer Science \\ Virginia Tech, Blacksburg, Va 24061 \\ lambros@vt.edu,perez@cs.vt.edu
}

\begin{abstract}
The development of the World Wide Web (WWW) and proliferation of web enabled devices have allowed various news agencies to enrich their traditional method of distribution of news through $\mathrm{TV}$, radio and print with simultaneous broadcast through the Web. The varying nature of devices through which the Web is accessed warrants different ways to feed the same content. This precipitates some variation in the way users interact with the news feeds. In this paper, we investigate how mental models and information scent affect this variation and user interaction on the whole. We present results from a preliminary survey conducted to capture the current news gathering behavior of general population and verify our assumptions. We then present observations from the study conducted using BBC news site over laptop, PDA and a cell phone.
\end{abstract}

\section{Author Keywords}

User Interaction variation, Multi-platform interfaces, news, news feeds, information scent, mental model.

\section{ACM Classification Keywords}

H.5.2 [User Interfaces]: Theory and Methods.

\section{INTRODUCTION}

With the proliferation of web enabled devices and continuous strides in WWW technology, today we can access the Web almost anywhere and anytime. Initially the desktop was our only portal into the Web hole but thanks to diverse web-enabled devices like PDAs, cell phones etc. we can now access the Web in various ways. With the availability of such web-enabled devices now designers have to design interfaces for systems across a pool of devices. Such interfaces are commonly referred to as multiplatform user interfaces [2]. As the device used to access the Web changes, so does our way of interacting with the Web (and the device). A user can use a keyboard and mouse when in front of a desktop/laptop computer and can perform highly interactive tasks while the same tasks might not be possible on a handheld PDA or a cell phone. Data entry becomes especially difficult on devices where the input mechanism is not highly efficient and convenient like cell phones and hence, tasks like filling out long forms do not translate well from one device with a larger form factor like desktops to another with a smaller form factor like PDAs or cell phones. However, tasks with no input, for e.g. browsing, and tasks with limited input like directed searching, translate better comparatively. Consequently, such tasks will find more acceptances among the user population across devices. Hence, the focus of this paper is on the latter kind of tasks.

Even if the type of tasks remains consistent across the devices, the way user performs the tasks will vary. As each interface is adapted for the device, the affordances incorporated and the structure of information will warrant a variation in user interaction. The objective of our research is to investigate such variation in user interaction. We look at two specific factors that might affect user interaction and effect variation: mental models and information scent. We first present results from a survey conducted to gauge current user behavior for gathering news and then present observations from our study conducted to investigate the effects of mental models and information scent.

Scott McDaniel [10] provides a very accurate description of mental models. A mental model is basically a simple mental image of the structure of the thing they represent and allows a user to predict results of his/her interaction. They just contain enough information to make accurate predictions based on what the user thinks is true. Studying mental models is important because they govern the way the user is going to use the system, which consequently depends on how well the user understands the system. A wellunderstood system with a good mental model will allow the users to use the system effectively. Moreover, as Don Norman [11] points out designer's view of the system might be different from user's view. He refers to the designer's view of system as design model and the user's view as user model. He argues that a designer should aim for compatible mental and design models for a more usable system. But this is only possible with a good understanding 
of the user's mental model and how it affects his/her interaction.

The effects of the difference between user's mental model of the system and design model are more pronounced in case of multi-platform user interfaces where the mental model might be different for different devices. A user's acceptance of using various devices to access the same system might depend on the adaptability and accuracy of his/her model. As the gap between designer's and user's view of the system is reduced, minimum effort will be spent on converting user intentions to actions and system outcomes to interpretations [9]. The user will find it easier to use the system and which will in turn spawn more frequent usage. So understanding how the mental models affect user interaction especially in the light of multiplatform user interfaces is the key to building effective and usable information systems across devices.

Information scent is a concept that been extensively researched at Xerox PARC by Peter Pirolli, Stuart Card, Ed Chi and others $[3,4,13,14]$. The concept of information scent was initially introduced by Pirolli and Card and is a refinement of a theory proposed by George Furnas [6] in which a representational object holds a "residue" of what hides behind it. Dr. Ed Chi [8] provides a good brief idea about information scent in one of his recent interviews: "Information scent is made of cues that people use to decide whether a path is interesting. These cues consist (of) images, hyperlinks and bibliographic citations related to the information needed." Such cues allow the user to calculate the probability of finding the right information for a particular path. In the absence of a valid mental model, users might use information scent to build one. Also there might be special cases, where the information scent for a path might be very strong but the path might not match the user's mental model and it will be interesting to observe user interaction in such cases. Through our research we explore the above issues and other effects of information scent on user interaction.

We chose news feeds as our domain since it is much more likely that in the near future, users will use the new webenabled devices to browse news. People today already get their news from multiple sources. They read news papers, listen to the radio, browse online and watch TV. As PDAs and cell phones become web enabled, many organizations, including news agencies, are starting to provide optimized versions of their desktop websites for these small form factor devices as well. In addition to optimizing news websites, new ideas are constantly being brainstormed to come up with innovative ways of feeding news content. RSS feeds [12] (also called webfeeds) are a good example of an innovative way to feed news content allowing the users to remain up-to-date with their favorite news sources. It is a standard format for syndicating news and news-like content. Most of the popular news agencies (CNN, BBC etc) already publish their news items through RSS feeds. A similar but more recent trend has emerged in the form of podcasting [7], which involves selectively downloaded audio feeds playable on iPod or iPod-like device. In the world of podcasting, a unit of news is called a podcast which is basically an audio file available through an RSS feed (It has a name, description and link to the audio file). Podcasts are nothing but online audio streams, which users can download selectively and sync it to iPod. It functions more like a TiVo for radio allowing the users to download whatever they want with the ability to listen whenever and wherever they want. As is evident, not only new ways of feeding news content are being invented but also new application domains for existing devices and how they can be applied to provide important news updates and items. What makes this domain special is that as users become more aware of multi-platform systems accessible over wide array of devices they are much more likely to use multiple devices for the purpose of news reading, since the entire domain involves tasks with no or little input (browsing and directed searching: categories we described earlier) and provides very important current and pertinent updates. For example, in case of podcasting, all the news items are available through iPod over touch of a button. Hence, the cost-benefit ratio is very high. As the users start becoming aware of various ways in which feeds are available (websites on PDAs \& cell phones, RSS feeds, Podcasts) and as the devices get cheaper and more affordable, we anticipate news sites to be among the first multi-platform information systems to gain widespread acceptance. The sudden popularity of Podcasting in a short span of time along with news agencies (BBC, NPR etc. ) recognizing the trend is evidence enough that users are willing to accept new forms of technology for news feeds. Therefore, horizontal usability among target devices, which is concerned with cross-platform usability requirements [16], becomes much more important in this domain.

\section{RELATED WORK}

Slone [17] conducted an interesting study about how user navigation and the use of web vary with the mental model, user experience with the web and user motivation. Our research is in the same vein, except that we are trying to tie user navigation with devices, mental model and information scent. A more relevant study was performed by Dr. Purush [15], where he tried to relate mental models with devices. He concluded that mental models are different for different devices but didn't have sufficient evidence to support the claim that a mental model is tied to a device. Don Norman suggests that mental model might be context dependent rather than device dependent. However, the claim by Don Norman is not supported by any study and is open to debate. As the mental model of the user changes with the device, user interactions with device might change and consequently the navigation pattern as well. A factor that might contribute to the mental model is the way user is required to interact with the device. Albers et. al. [1] state that users have an active mental model of all sites and they evaluate the events against that mental model. If the mental 
model is incorrect, then the information retrieved from the system might not be correctly interpreted giving rise to incorrect actions. Eventually the user will shift to a different mental model but not till data interpretation problems have reached a critical stage. Arguably, we can say that mental models are based on interaction and they govern future interactions as well. If we take the same argument and factor in different devices, it paints an interesting picture about mental models, devices and the way information is presented. If the information presented on a particular device coincides with user's mental model, the user might not shift the mental model from device to device. But if the information presented doesn't coincide with user's mental model, then the user has to rely on the combination of mental model and the "information scent" presented in the data. If he still gets the task done, then it implies that the information had sufficient "scent" to guide the user through the task.

The concept of information scent is based on Information Foraging Theory, which according to Peter Pirolli [13], "addresses how people will adaptively shape their behavior to their information environments and how information environments can be best shaped to people". The specific aspect of Information Foraging Theory that we will be examining is the information scent of web pages, which refers to the cues used by users to make decisions related to the selection of information sources to consume. We use an expanded definition of information scent that includes the cues used by users to determine the purpose of structures within a site. For example, in our study we discuss the problems with a drop-down list widget, which users had problems locating, or understanding its purpose.

There are numerous other ways that can be used to measure information scent which are relevant to our research. Card, Pirolli et. al. [3] have developed a variety of methods for usability evaluations based on information scent. One of these is a method for visualizing web navigation paths using web behavior graphs, which include information about the information scent of each page. With these graphs, developers can evaluate the usability of a website and relate it with the information scent of each page. With their method, information scent is measured in two ways: 1) User comments during browsing such as "that looks promising" or "I think I'll try something else" are used as indicators of high or low scent and 2) Side experiments take place with expert judges who rate the information scent of pages with respect to a given task.

Other related work includes SNIF-ACT [14], a system which simulates a user searching for information online. SNIF-ACT uses a cognitive model, based on Information Foraging Theory, to rank links' relevance to the information need. In experiments with real users, a significant matching pattern between the users' choices and SNIF-ACT's predictions was found. Other computational methods have been developed for understanding the relationship between user needs and user actions [4].

\section{PRELIMINARY SURVEY \& RESULTS}

A preliminary survey was conducted to gauge user behavior for gathering news with respect to various sources used and the manner in which they are used. A survey was designed in paper and web format and distributed across listservs and discussion forums at Virginia Tech. We received 15 responses to the survey and base our observations on those responses.

Web (87\%), TV (73\%) and Radio (60\%) were identified as the most important sources for everyday news by most of the respondents. Almost all of the respondents (over 90\%) who watch TV for news do so in the evening or night in the bedroom or living room $(\sim 94 \%)$. On the contrary those who use radio, do so mostly in the morning (over 90\%) commuting to work in car $(81 \%)$. All the users who use web for news do so from office $(100 \%)$. This reinforced our choice of using news feeds as domain of investigation since most of the users are already familiar with the concept of online news feeds and browse the feeds almost daily. Also they currently get their news from multiple sources using multiple devices and hence, will be more receptive to the idea of using multiple devices for browsing news.

Most desktop users browse the sites regularly for news headlines $(67 \%)$ or without any specific article in mind (60\%). At least sometimes, most users (87\%) also look for a specific news item while browsing through a desktop / laptop. Respondents preferred to use desktop computers whenever they had to view multimedia rich websites or wanted to provide some kind of input. This again confirms our assumption that users will not use web enabled devices with smaller form factor with poorly developed input mechanism for highly interactive tasks.

Encouraged by the survey results which confirmed our assumptions we went ahead with the study to explore user interaction issues involving mental models and information scent in detail.

\section{THE STUDY}

We had 8 participants for the study organized in four groups. Each group used two devices for the study.

\begin{tabular}{|c|c|c|c|}
\hline Group & Participants & \multicolumn{2}{|c|}{ Devices used (in order L to R) } \\
\hline 1 & $1 \& 2$ & Laptop & PDA \\
\hline 2 & $3 \& 4$ & PDA & Laptop \\
\hline 3 & $5 \& 6$ & PDA & Cell phone \\
\hline 4 & $7 \& 8$ & Cell phone & PDA \\
\hline
\end{tabular}

Table 1. Participant distribution for the study

The study was performed using the BBC News site which is available in HTML format (for desktop and PDA) and in WAP format (for the cell phone). An Averatec laptop with a resolution of 1024 X 768 on a 12" screen , a Palm Tungsten C with a resolution of 320 X 320 and a Sony 
Ericsson T610 phone with a resolution of 120 X 160 were used for the study. Each group was given four different tasks from the following list of tasks, each one on a separate sheet of paper:

1. Find the article with the headline: headline

2. Find the following article: description.

3. Find any two headlines from a section

4. Select any two headlines on one device and locate those headlines on the second (Groups $3 \& 4$ )

5. Find out what BBC news viewers have to say about a current event (Groups 1 \& 2).

Regarding Task \# 5, the opinions of the viewers are organized in a section called "Have your say" (available on PDA and laptop). The section can be accessed in two ways from the desktop (Main menu and through the article) and only one way from PDA (Main menu).

The participants had to perform these tasks using two different devices in the order (for devices) described in Table 1. The order of tasks itself was random among the participants but was consistent for a particular participant across devices. The tasks were a mix of directed searching and browsing. Task \#4 is an example of browsing on one platform translating to directed search on another. Task \# 2 is an example of an article viewed earlier but a user can't remember the name of the article. $\mathrm{S} /$ he just remembers the content but not the headline. Task \# 1 is an example where the user remembers the exact headline. Task \# 5 is interesting since paths followed to reach the desired item can be different on different devices. Task \# 3 is a case of plain browsing.

The participants were asked to think out loud and a facilitator took notes. All the sessions were recorded with a video camera focused on the device screen for reviewing and analysis. After completion of the tasks over each device, each participant was asked to fill out a questionnaire, which gave us an idea about the mental model s/he had developed for the system over that device. The questionnaire also had questions, which helped us evaluate information scent of links they followed. In addition to the questions, we also independently reviewed the links followed by the participant using the recorded sessions and rated the information scent for links \& structures as good or bad, depending on how the participant felt following the link (based on what $\mathrm{s} /$ he said when $\mathrm{s} / \mathrm{he}$ was thinking aloud), his/her confidence in following the link and his/her satisfaction with the result. This method of evaluating information scent is similar to Pirolli's and Card's [2] method with the exception that we did not produce web behavior graphs from our measurements and we only evaluated the links that the users followed.

We present our findings in three separate sections. The first section describes general usability issues. In the second and third section we discuss observations related to information scent and mental models respectively.

\section{GENERAL USABILITY ISSUES}

This section describes some general usability issues that were caused by the differences in the various versions of the site. In particular, we discuss usability problems related to the PDA's rendering of the drop-down menu and how designers can avoid such problems.

\section{Menu drop-down list on the PDA version}

At the top left corner of the PDA front-page, there is a drop-menu which contains links to the site's various sections [Fig. 1]. The BBC News site is organized into sections by geographical regions. This drop-down list is effectively the site map and its use was highly linked to the participants' navigation path and to the effort needed by participants to complete their assigned tasks.

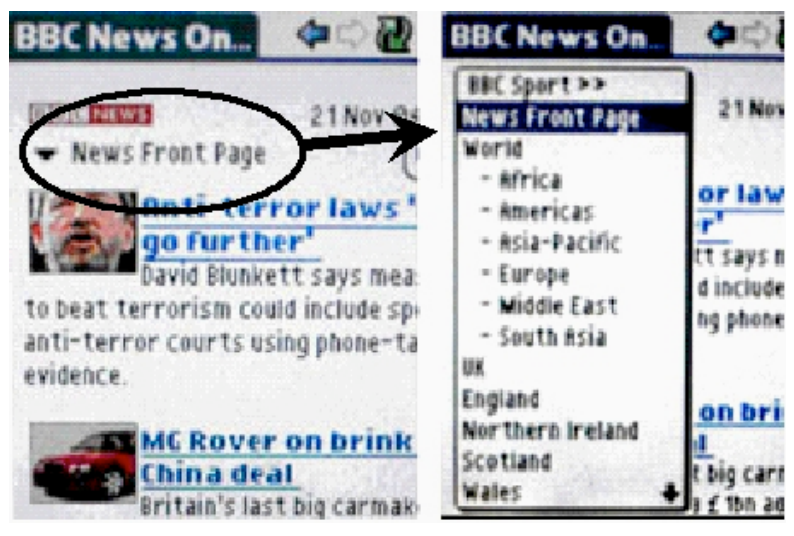

Figure 1: Main Menu drop-down list on PDA

The problem we encountered was that either most participants could not find it, or did not understand that it was the Main Menu containing links to the site's sections. Most of the participants did not use it on their own, and they had to be informed about the menu and its purpose, so that they could complete some tasks that could not be finished otherwise. The rest relied on a set of links, which could be found at the bottom of the front-page, to navigate to other sections. We believe that the presentation of the menu as a drop-down list results in usability problems for two reasons:

The widget was poorly rendered by the built-in browser. On most popular desktop internet browsers, a drop-down list is rendered as a widget with a solid border, containing some text (usually the first item in the list) and a raised button with a downwards pointing arrow symbol on the right of the text. This type of graphical layout implicitly tells users that they can manipulate the widget by clicking on the button. Even if users fail to realize this intentionality, the fact that this layout has been used consistently and almost universally to signify a drop-down list has made experienced users understand its meaning.

On the other hand, the PDA rendered the drop-down list as the first item on the list with an arrow symbol on its left, without the standard border, and without any type of button 
widget to contain the arrow. This rendering made it difficult for participants to understand that the widget was a dropdown list.

There was no graphical or textual description of the purpose of the widget.

The drop-down list, as we said before, displays the first item on the list by default. In this case, the first item was "News front page". While the front-page is indeed a section of the site, the users did not realize that the widget served as a site map containing all the sections. A better design would either have some kind of description above or on the left on the drop-down list, or would have a dummy selection as the first item, which described the list itself, as many websites do today.

Improper display of subsections on PDA \& Cell Phone On the desktop version of the BBC News site, the Main Menu lists "UK" as a main section. Selecting that section results in UK's regions (Wales, Ireland, etc.) and subcategories (Politics, Education, etc.) being listed separately, on a darker background and under "UK" in bold to indicate that these regions and subcategories are subsections of the UK section. When "UK" is selected, a selection of the top stories, organized by regions, is displayed. To find all articles for a particular subsection, the user would have to click on the corresponding link, which could be found on the aforementioned site map or above the articles pertaining to that subsection.

However, on the PDA and cell phone versions of the site, UK and its subsections are listed concurrently, without any differences in font, indentation or color. See Fig. $1 \& 2$ for comparison. Also, in the UK section, links to the corresponding subsections are not provided above the grouped articles.

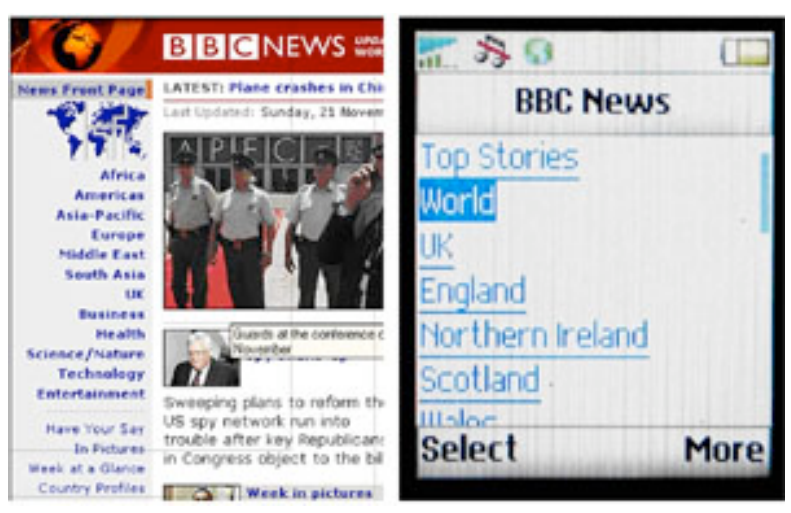

Figure 2: Main Menu on Desktop and Cell Phone

When users were asked to find articles related to England or Scotland, most of them selected the UK link first, without noticing on the PDA and cell phone versions that there were links for England or Scotland. On the desktop, this was not a problem because they found the links to the subsections within the UK section. However, on the PDA and the cell phone, since only the selection of headlines was shown, and there were no links to the subsections, they could not find the articles. Furthermore, since they did not notice the subsection links, they assumed that the UK section contained all of the articles that pertained to the UK and therefore were frustrated believing that the articles they were assigned to find were not there.

Therefore, the menu gave users misleading information about the content and organization of the UK section and its subsections. The information that the users were expecting to find was not available through the sections they chose on the menu. For this reason, the site map on the PDA and cell phone versions of the BBC News page has bad information scent when the user task is related to news articles contained in UK's subsections.

From these observations, we can conclude that when designers are developing interfaces for multiple platforms, they need to check that the widgets are rendered properly across the target devices. If these widgets do not provide good scent on some devices, then the designers must make sure they provide some additional information for those widgets to supplement the information scent on those problematic devices.

\section{OBSERVATIONS RELATED TO INFORMATION SCENT}

In this section we shall discuss some of the interesting observations we made, from an information scent point of view, in particular, general findings about information scent and its relation to the users' decision making process.

\section{Using nouns in links improves information scent}

We observed that users extracted one or two nouns (especially proper nouns) from their task descriptions and used them to identify and choose links. If the link that they were looking for did not contain the nouns they had in mind, they did not notice it.

For example, one task was stated as "Find out what BBC News viewers have to say about [a current event]". Most participants either tried to find a news article related to that event, or looked for some kind of link that had "BBC Viewers" in it. The fastest way to achieve the task would be to select the "Have your say" link on the site map, which would result in displaying the section where readers' comments are submitted and displayed. However, the wording of the link (lack of strong or proper nouns) did not help participants quickly associate their thoughts with that section's contents, and therefore most users did not employ that link on their first try, even though the task description also included the phrase: "have to say".

This finding possibly indicates that a headline's information scent increases when more proper and/or descriptive nouns are included in it. By including such nouns in headlines, users' ability to find a previously read article is enhanced when they move from one device to another, since they use proper nouns as the primary filter and verbs as the secondary filter. 


\section{Scent Overload}

Another trend we noticed during our study is that participants often did not notice the links they were looking for, when those links were located in a section crowded with other links. For example, on the PDA version, as described earlier, an alternate site map is provided at the end of each page. This site map consists of multiple lines of short links. Each link by itself has good information scent but the fact that there are so many of them crowded together, it effectively reduces the scent of each one. We frequently observed participants looking at that site map, stating that they were looking for a link that indeed was there, yet they did not find it and moved on. Crowded hyperlinks are known to be a problem for users with disabilities [5] but here we see that because of their inherent information scent overload, they can be problematic for any type of user. This problem arose because the designers migrated the style of links at the bottom of the page directly from the desktop/laptop version to the PDA without considering the form factor of the PDA. As we discuss later in the paper, the information structure across devices should be consistent but at the same time it should be comprehensible as well. This is another example of failure of presenting comprehensible structure.

\section{Information Scent vs. Amount of Work}

All participants that browsed the desktop and/or PDA versions of the news site, always attempted to find the articles specified in their tasks by scanning the front-page first. Even on the desktop where the site map is clearly displayed on the left, participants chose to ignore it until they finished looking through the front-page. We believe this behavior can be explained by the following two theories:

1. The concept of the front-page has strong information scent in itself. Users view the front-page as a place where any important article is probably located.

2. Users will typically choose the path that requires less work, even if the information scent is stronger on a different path. Scanning the front-page is not a big risk for them since they'll remain on the same page and avoid moving deeper into the site and getting lost. Also, the cost of undoing such an action is much lesser than that of following an erroneous path that would lead them deeper into the site. The potential benefit of finding the article in the front-page offsets the cost of scanning it. This theory can be further supported by the participants' behavior on the cell phone site: The cell phone version does not have a front-page with articles. It simply contains links to other sections, including a section called "Top Stories". The participants who browsed on the cell phone never selected the "Top Stories" link but rather selected the link with the highest information scent, relative to their task at hand.

We can therefore conclude that when the perceived amount of work for each potentially rewarding path is equal, users will always follow the path with the strongest information scent. On the other hand, if one of those paths has less information scent but a potentially high cost-benefit ratio, they will probably follow that one even if it goes against their mental model. This conclusion is of particular importance for devices which have a capability of displaying a "Front page" or a similar concept.

\section{OBSERVATIONS RELATED TO MENTAL MODELS}

Users preserve the mental model when they move from one device to another.

This is the single biggest conclusion drawn from our observations. There were various instances to support our conclusion. For the task involving finding out viewer comments, Participant \#2 after an initial struggle, found the "Have Your Say" section as a separate section from the Main Menu over laptop. S/He used the same model over PDA and found the section within 13 seconds. Participant \# 3 found the "Have your say" section once s/he browsed to the article and then followed the link to the section from the bottom of the page. Hence, according to his/her mental model the section was specific to the article and not available at the higher level of menus. The participant simulated a similar browsing pattern on the laptop as well, although there was clear "Have your say" section link in main menu. The link was overlooked because according to the user's mental model, the information was stored inside the article and available only through it.

Users use information scent to build their mental models. When the participants were unaware of the location of article within the site, they used information scent to guide them through to the article. Once they found the article they built a mental model of the location. In other words, a successful completion of the task finalized the user's mental model for the information at hand. Hence, information scent plays a very important role in building a correct model with the highest accuracy for finding the required information. We draw this conclusion from numerous observations. Whenever participants failed to find the article on the frontpage (for laptops and PDAs) or when they were starting their search on cell phones for a specific article, they categorized the article according to region or category (like sports, business etc.) or both and used the information scent of the links to guide them through. For example, Participant \# 7, for the headline "Indonesia Spied on Australians", used "Indonesia" to look for the article in "Asia-Pacific" section. Other participants exhibited similar behavior.

Use of Proper nouns in links aids building mental models. Participants used Proper Nouns from article headlines or task descriptions as their primary search criteria and then used verbs as secondary filters. When they visually scanned an article they picked up the proper nouns before anything else, and this helped them in categorizing or understanding what the article was about. As we argue in another section, using such nouns in links lends more scent to the link and in turn makes it easier for the user to build the mental model. 
A headline such as "Missing mother reservoir search", doesn't have any proper noun and makes it difficult for a user to locate the article.

\section{Mental model changes across devices only for the fractured part.}

As argued previously, users preserve the mental model when they move from one device to another. Their mental model is reinforced if the task is completed successfully and the required information is obtained. However, in cases where the information is not found by following the same path as on previous device, they repair the mental model or in other words "adapt" the mental model for that particular information and for that particular device. For example, Participants \# 1 and 2 were given a task where the article was available on the front-page over the laptop and was NOT available at all over the PDA. As expected they found the articles easily on the laptop. Since they carried the mental model to the PDA, they expected to see the article on the front-page on the PDA as well. However, once they couldn't find the article, they used nouns ("Turkey" in this case) to categorize it by region and used information scent to locate the article. They couldn't find the article on the PDA and correctly concluded that it's available on the main page on the laptop and unavailable over the PDA. Similarly, Participant \# 1 found the "Have Your Say" section through the article over the laptop and had a model of accessing the viewer comments through the article. However, for the PDA, the participant was unable to duplicate the model and refined it to conclude that the section can be accessed only through the Main Menu on the PDA. The rest of the model remained the same for the participant.

\section{Strong information scent takes precedence over mental models}

Although users preserve the mental model across devices and try to follow the same path, there are special cases where when presented with a link with a very strong information scent, they choose to follow it against their mental model. For e.g. Participant \# 7 located a headline about sports under "World $->$ South Asia" section on the cell phone. However, on the PDA, the user browsed to World section and then went to "Sports ->South Asia" for the same headline. This is because the article s/he was searching for was related to sports and the participant thought $\mathrm{s} /$ he had more chances of easily finding the article under this section. However, when asked to draw a mental model of the organization of the article within the site, the participant drew a similar organization in both cases. This brings us to the next conclusion.

In cases where the users decide to follow a different path due to following a link with stronger information scent, mental models of the overall organization remain unaffected. Another observation, which supports our conclusion, is from Participant \# 3. We have already explained how the concept of information scent applies to links as well as to overall structures/concepts. $\mathrm{S} / \mathrm{He}$ found the article about
Nigeria under "World - > Africa" on the PDA. Although the participant was fairly confident of locating the article in the same section over the laptop, s/he decided to first scroll through the front-page because, as a concept, "front-page" offers a stronger scent of locating an article with a current headline and, in the case of success, offers faster results.

Mental model depends on information structure as well as the presentation of information structure and not on the device. Also, the information structure should follow a logical pattern.

As we have already seen, the mental model is consistent across devices and the factor that contributes the most to this consistency is the consistency of the structure of information and a comprehensible presentation of that structure. By comprehensible presentation we don't mean that the presentations over various devices should be "rendered" in the same way. For example, on the BBC website, the menu on the left on the laptop shouldn't be rendered in the same way on the PDA. It is ok to render it as a drop-down list, however as discussed earlier in the paper, this list was not easily comprehensible, as it didn't have the necessary "scent" of a menu. Hence, initially the users were struggling more on the PDA than laptop and cell phone to get a picture of overall structure. Also, one interesting aspect was that news articles for Wales, Scotland, etc. were organized under UK section on the laptop, while they were separate sections on the same level as UK on cell phone and PDA. This had considerable impact on the participants' understanding of the system. When prompted to get news about Wales or Scotland, they tried to go in to UK section. Even those participants who didn't use the laptop and hence, were unaware of the information structure on the laptop tried to access UK section first but quickly found the links to this section below them. This shows that information should be organized in a logical manner and should follow consistent pattern across devices. This will greatly ease the burden of building and adjusting mental models on the user since the structure of information will be the same and easier to remember because of a logical pattern. Purush et. al. propose a question about the dependency of mental models. We conclude that the mental models are not devicedependent (as already seen by our previous conclusions). They depend more on the structure of information presented to the user on the device and it is possible to have just one mental model across devices if the consistency in information structure is maintained and is presented in a comprehensible manner to the user. This is in direct contrast to Purush's conclusion that mental models are different for different devices. Mental models are different only for the fractured part where the information structure doesn't match the mental model from previous device.

User's mental model assumes that all the presented links are "accessible" on that device.

We ran into a problem with Participant \# 3 while browsing the $\mathrm{BBC}$ news site. It had a link to Zimbabwe's government 
website which was not optimized for PDA viewing and the participant accessed it unintentionally. The loading time was greatly increased and the participant expressed lot of frustration during the interval. At the end of the loading, the participant wondered either something was wrong with the page or the designers had made a mistake since the page had too much of horizontal and vertical scroll. We concluded that users expect links to point to only those sites which are optimized to be viewed on that device. A break in this model causes lot of frustration and eventually the user might be wary of browsing the links on the website for the fear of clicking on a wrong link.

\section{CONCLUSION AND FUTURE WORK}

We have presented few interesting observations and conclusions about how mental models and information scent affect the user interaction especially in case of multi platform news feeds. We hope the designers of future multiplatform news feeds and multi-platform user interfaces in general, will be able to use some of the conclusions and recommendations presented for building better systems. We noted an interesting thing about the way users represented their mental models. Two participants represented mental models for devices with larger form factor as vertical and for devices with smaller form factor as horizontal, although the organization of the information remained the same. The reasoning behind the variation in organizing the mental model might be that users think of information systems as sequential entities on smaller form factor devices while as a hierarchical entities on larger form factor devices. It is an interesting observation but requires more investigation.

In future, we would like to conduct the study over a larger data set in order to reinforce our claims and observations. The interfaces used in our studies were visual in nature. It will be interesting to explore similar issues when one of the interfaces is a uni-dimensional sequential feed like a voice interface. The trend of Voice portals is beginning to gain momentum and development of standards like VoiceXML is evidence enough that the trend is being recognized. In the future, we plan to replace one of the interfaces with a VoiceXML interface and conduct similar kind of study. In the same vein, VoiceXML interface can be replaced by a podcast to study some other interesting aspects. With voice interfaces, another aspect of mental models can be evaluated: the effect of presenting the visual before the audile and vice versa.

\section{REFERENCES}

1. Albers, Michael J., Kim, Loel. User web browsing characteristics using palm handhelds for information retrieval. In: Proceedings of IEEE professional communication society international professional communication conference and Proceedings of the 18th annual ACM international conference on Computer documentation: technology \& teamwork, 2000, 125-135.
2. Ali, Mir Farooq, Pérez-Quiñones, Manuel A., Building Multi-Platform User Interfaces, Multiple User Interfaces, John Wiley \& Sons, 2004.

3. Card, Stuart K., Pirolli, Peter, Van Der Wege, Mija, Morrison, Julie B., Reeder, Robert W, Schraedley, Pamela K., Boshart, Jenea. Information Scent as a Driver of Web Behavior Graphs. Proceedings of the SIGCHI conference on Human factors in computing systems, March 2001, 498-505

4. Chi, Ed H., Pirolli, Peter, Chen, Kim, Pitkow, James, Using information scent to model user information needs and actions and the Web, Proceedings of the SIGCHI conference on Human factors in computing systems, Seattle, Washington, March 2001, 490-497.

5. Cook, Robin A., Gladhart, Marsha A., A survey of online instructional issues and strategies for post secondary students with learning disabilities, Information Technology and Disabilities (Online Journal) Vol. 8, No. 1, January 2002. http: //www.rit.edu/ easi/itd/itdv08n1/cook_gladhart.htm

6. Furnas, George, Jul, Sussane, Navigation in Electronic Worlds, Workshop Report, CHI, 1997.

7. Gahran, Amy. What is Podcasting and Why Should You Care? http://blog.contentious.com/archives/2004/ 10/29/what-is-podcasting-and-why-should-you-care.

8. Gonzalez, Angel. Hot on the Scent of Information. http://www.wired.com/news/technology/0,1282,44321, 00.html.

9. Hutchins, Edwin, Hollan, James, Norman, Don, Direct Manipulation Interfaces, User Centered System Design : New Perspectives on Human Computer Interaction, Laurence ErlBaum Associates Publishers, 1986, Chapter 5, pp 87-124.

10. McDaniel, Scott. What's Your Idea of a Mental Model?http://www.boxesandarrows.com/archives/what s_your_idea_of_a_mental_model.php.

11. Norman, Don, Cognitive Engineering, User Centered System Design : New Perspectives on Human Computer Interaction, Laurence ErlBaum Associates Publishers, 1986, Chapter 3,pp 31-65.

12. Pilgrim, Mark. What is RSS? http://www.xml.com/pub/a/2002/12/18/dive-intoxml.html.

13. Pirolli, Peter, The Use of Proximal Information Scent to Forage for Distal Content on the World Wide Web, Working with Technology in Mind: Brunswikian Resources for Cognitive Science and Engineering, Oxford Press, 2004.

14. Pirolli, Peter and Fu, Wai-Tat. SNIF-ACT: A Model of Information Foraging on the World Wide Web. Ninth International Conference on User Modeling, Johnstown, PA., 2003, 45-54. 
15. Purush, Avi, The Measurement and Comparison of Cross-Device Mental Models, Human Oriented Technology Lab, Carleton University, 2004. http://www.carleton.ca/hotlab/hottopics/Articles/OnMe ntalModels.html.

16. Seffah, Ahmed, Javahery, Homa. Multiple User Interfaces, John Wiley \& Sons, 2004, Chapter 2, p. 16.
17. Slone, Debra, The Influence of Mental Models and Goals on Search Patterns During Web Interaction, Journal of the American Society for Information Science and Technology, Vol. 53 , Issue 13, November 2002, 1152 - 1169 . 